\title{
Opinion
}

\section{The Burden on Graduate School Recommenders}

At this time of year, many of us will be assisting our undergraduate and master's students in applying to graduate school. We will also be helping our new PhDs apply for postdoctoral or tenure-track positions. While both application processes require letters of recommendation, they are otherwise quite different. Where the second requires us simply to upload the letter to MathJobs, the first often forces us to negotiate a wide variety of graduate school application systems with a plethora of extra questions and check boxes. This can be frustrating after one has spent time writing an informative, detailed letter covering all aspects of the applicant's qualifications. In some cases, the additional questions ask for evaluations of the applicant's originality, creativity, research potential, etc. If you are lucky, the answers to such questions use check boxes; if you are unlucky, entire paragraphs are demanded. We believe that we must push back against the unreasonable demand on our time from these extra recommendation forms, which likely provide only marginal value to graduate programs.

A simpler approach should be possible. Why do we trust our colleagues to recommend new PhDs or postdoctoral fellows with nothing but a letter, while for graduate applications we require that same information again, in a somewhat different format? In our experience, when we evaluate student applications, there is usually little if any extra information not in the letter. And the ratings provided are typically more inflated than they are in the letter. Of course: we all realize that providing an applicant with anything but the strongest endorsement to a question like "Do you support the student's application?" is a big strike against them. In a letter we can be more nuanced and offer more perspective.

What can be done? Different solutions are possible. Some require more effort than others, but may have a larger impact. For starters, all of us should examine the letterof-recommendation process of our own departments. If you find the process annoying and too time consuming, chances are so will everyone else. If so, push for a change.

Recently, we have been inspired by a note Robert Strichartz wrote at the end of a letter of recommendation one year ago, which was read by the first author. Professor Strichartz has given us permission to reproduce the note here. Verbatim, it reads:

If your application form asks for ratings of the student in various categories I will either deliberately leave them blank, or if your system does not allow the option of leaving them blank I will check the highest rating in all categories. In addition, the department administrative staff is designated to submit the application material for me. If your system requires other information in regards to my background, it may not be accurate. In both cases please disregard the ratings and faculty background information. I understand that this information is used in your evaluation process; however, I would like to suggest that you consider modifying your system to allow the option to skip ratings and background sections to make the process more efficient.

The inclusion of this note did not affect the standing of the applicant, which is of paramount importance. We have adopted this approach in our own letters this application season. Over the long term, it would be helpful to have a central system for letters of recommendation that would be used by all graduate programs in mathematical fields, something like MathJobs. Letters would be uploaded once for each applicant, after which the applicant decides which department receives which letters. The AMS, with its MathJobs experience, and its central position in the mathematical sciences, seems well placed to develop such a system. The rest of the application materials would not be entered into this online system but instead would be submitted by whatever means various departments currently use. Medical schools provide an example: 139 of the 141 accredited US medical schools receive letters of recommendation for more than 45,000 applicants through a website run by a professional society (services.aamc.org/letterwriter/).

The current systems for submitting letters of recommendation for graduate school applicants are unnecessarily burdensome to students' recommenders. Technology and coordination could ease the burden, as could simply more careful consideration to the amount and type of information that is truly needed. We should start by ensuring that the system at our home institutions is not one of the culprits. Beyond that, as recommenders we can follow Strichartz's idea of refusing to fill out additional forms, which might over time lead to departments changing their approach to a more reasonable one.

- Bernard Deconinck University of Washington bernard@amath.washington.edu -Jan Medlock Oregon State University jan.med1ock@oregonstate.edu 